\title{
DISPONIBILIDADE DE HORAS DE FRIO E SUAS RELAÇÕES COM TEMPERATURA PARA TRÊS LOCALIDADES DE MINAS GERAIS
}

\author{
Vinicius Evangelista Silva ${ }^{1 *}$, Maria José Hatem de Souza ${ }^{2}$, Gilciano Saraiva Nogueira ${ }^{3}$, \\ Aristides Ribeiro ${ }^{4}$, Fernando Palha Leite ${ }^{5}$
}

\footnotetext{
${ }^{1}$ Especialista em Nutrição e Manejo Florestal da Eldorado Brasil Celulose - Tres Lagoas (MS) e Doutorando em Sistemas de Produção pela UNESP, Universidade Estadual Paulista (UNESP), Faculdade de Engenharia, Câmpus de Ilha Solteira - SP. Email do autor correspondente: viniciusesilva@ yahoo.com.br

2 Professora de Meteorologia e Climatologia, Departamento de Agronomia, Universidade Federal dos Vales do Jequitinhonha e Mucuri - Campus JK - Diamantina, MG.

${ }^{3}$ Reitor da Universidade Federal dos Vales do Jequitinhonha e Mucuri - Campus JK - Diamantina, MG.

${ }^{4}$ Professor Meteorologia Agrícola, Departamento de Engenharia Agrícola, Universidade Federal de Viçosa, Viçosa - MG.

${ }^{5}$ Coordenador de Tecnologia Florestal da Empresa Celulose Nipo-Brasileira S. A. Ipatinga, MG.
}

RESUMO: Em várias localidades não existem registros horários de temperatura do ar, o que dificulta a elaboração de vários estudos sobre a aptidão agrícola de várias espécies e cultivares. $\mathrm{O}$ objetivo do presente trabalho foi determinar por meio de registros horários de temperatura do ar o número de horas de frio (NHF) inferiores a $7^{\circ} \mathrm{C}, 10^{\circ} \mathrm{C}$ e $13^{\circ} \mathrm{C}$ em três localidades de Minas Gerais, e correlacioná-las com a temperatura mínima e máxima média mensal para identificar as potencialidades dessas variáveis em estimar o NHF. Utilizaram-se para este estudo dados horários de temperatura do ar dos municípios de Belo Oriente, Antônio Dias e São Domingos do Prata - MG, durante o período de junho de 2001 a dezembro de 2007. A localidade de Antônio Dias apresentou os menores valores de temperaturas do ar e maior número de horas de frio, quando comparada com as demais localidades, enquanto que a localidade de Belo Oriente apresentou as maiores temperaturas e o menor número de horas de frio. As variáveis temperaturas médias mensais mínima e máxima do ar correlacionaram-se bem com o $\mathrm{NHF}<13{ }^{\circ} \mathrm{C}$ em todas as localidades, não acontecendo o mesmo com o e o NHF $<10^{\circ} \mathrm{C}$ e $\mathrm{NHF}<7{ }^{\circ} \mathrm{C}$ que apresentou valores muitos fracos ou não significativos de correlação. As variáveis temperatura mínima e máxima médias mensais do ar podem ser utilizadas para estimar o NHF $<13{ }^{\circ} \mathrm{C}$.

Palavras-chave: Temperatura do ar. Frio hibernal. Indices bioclimáticos.

\section{AVAILABILITY OF COLD HOURS FOR THREE LOCALITIES OF MINAS GERAIS}

\begin{abstract}
In several localities, there are no hourly air temperature records, which makes it difficult to prepare several studies on the agricultural suitability of various species and cultivars. The objective of the present study was to determine the number of hours of cold (NHF) below $7^{\circ} \mathrm{C}, 10^{\circ} \mathrm{C}$ and $13^{\circ} \mathrm{C}$ in three regions of Minas Gerais by means of hourly air temperature records and to correlate them with the minimum and maximum average temperature To identify the potentialities of these variables in estimating the NHF. The air
\end{abstract}

Cultura Agronômica, Ilha Solteira, v.26, n.4, p.560-572, 2017 
temperature schedules of the municipalities of Belo Oriente, Antônio Dias and São Domingos do Prata - MG, during the period from June 2001 to December 2007, were used for this study. The Antônio Dias region presented the lowest values of air temperatures and higher number of cold hours when compared to the other localities, while the Belo Oriente region presented the highest temperatures and the lowest number of cold hours. The mean minimum and maximum monthly air temperatures correlated well with the NHF $<13{ }^{\circ} \mathrm{C}$ and the in all locations, not the same with the NHF $<10^{\circ} \mathrm{C}$ and the NHF $<7^{\circ} \mathrm{C}$ that presented very weak relationships or non-significant values of correlation. A minimum and maximum monthly air temperature variables can be used to estimate NHF $<13^{\circ} \mathrm{C}$.

Key words: Temperature of the air. Winter Cold. Bioclimatic index.

\section{INTRODUÇÃO}

A quantificação do número de horas de frio torna-se referência para determinar a aptidão agrícola de algumas espécies/cultivares para uma determinada localidade. Para a quantificação do período de resfriamento é normalmente utilizado o índice bioclimático, genericamente denominado de "Número de Horas de Frio" (NHF), característico para cada espécie e cultivar (CARDOSO et al., 2012; MORAIS; CARBONIERI, 2015).

Para mensurar o frio necessário para quebrar a dormência e ocorrer a brotação, o método mais utilizado é o somatório diário do número de horas com temperaturas abaixo de $7^{\circ} \mathrm{C}$ ou abaixo de $10^{\circ} \mathrm{C}$ durante o período de outono e inverno respectivamente (SOUZA et al., 2009). Esse limite, entretanto, não é aplicado a todas as espécies e cultivares, sendo também utilizado o período em que a temperatura do ar permanece abaixo de $13^{\circ} \mathrm{C}$ para caracterizar espécies menos exigentes em frio invernal (RIBEIRO et al., 2006). Tal método tem sido o mais utilizado e difundido pela simplicidade de cálculo (SILVA et al., 2002).

Muitas vezes, a ausência de um banco de dados horário de temperatura do ar impossibilita a quantificação do NHF, tornando-se indispensável estabelecer outras relações mais simples para quantificá-los (CARDOSO et al., 2012). Diante disso, outra forma simples para obtenção do NHF é a partir de variáveis mais fáceis de serem obtidas, como por exemplo, os valores médios mensais das temperaturas mínima e máxima as quais possibilitam a elaboração de modelos matemáticos para quantificação do NHF.

Além disso, a localidade do Vale do Rio Doce em Minas Gerais é considerada como apta para cultivo de café e de algumas espécies de frutíferas (ASSAD et al., 2004), porém são ainda incipientes trabalhos exploratórios sobre o número de horas de frio, bem como a sua disponibilidade para esta região (CARDOSO et al., 2012).

Diante o exposto, o objetivo deste trabalho foi correlacionar a variável NHF com a temperatura do ar em três localidades de Minas Gerais, e através das estimativas verificar se as localidades avaliadas possuem aptidão para o cultivo de espécies de clima temperado.

Cultura Agronômica, Ilha Solteira, v.26, n.4, p.560-572, 2017 


\section{MATERIAL E MÉTODOS}

Utilizaram-se para este estudo dados obtidos em três estações meteorológicas automáticas pertencentes a Empresa Florestal Celulose Nipo-Brasileira (CENIBRA), localizadas nos municípios mineiros de Antônio Dias, Belo Oriente e São Domingos do Prata. Os registros climáticos são referentes ao período de junho de 2001 a dezembro de 2007. Os dados das coordenadas geográficas de cada estação automática e suas respectivas classes climáticas segundo Köppen (ALVARES et al., 2013) são apresentados na Tabela 1.

Tabela 1. Coordenadas geográficas e altimétricas das estações automáticas e classificação climática segundo Köppen dos municípios de Belo Oriente, Antônio Dias e São Domingos do Prata

\begin{tabular}{ccccc}
\hline Local & $\begin{array}{c}\text { Latitude } \\
\text { (graus })\end{array}$ & $\begin{array}{c}\text { Longitude } \\
\text { (graus })\end{array}$ & $\begin{array}{c}\text { Altitude } \\
(\mathbf{m})\end{array}$ & $\begin{array}{c}\text { Classe } \\
\text { Climática }\end{array}$ \\
\hline Antônio Dias & $-19^{\circ} 29^{\prime}$ & $-42^{\circ} 52^{\prime}$ & 1.273 & $\mathrm{Cwb}$ \\
Belo Oriente & $-19^{\circ} 17^{\prime}$ & $-42^{\circ} 23^{\prime}$ & 233 & $\mathrm{Aw}$ \\
$\begin{array}{c}\text { São Domingos do } \\
\text { Prata }\end{array}$ & $-19^{\circ} 46^{\prime}$ & $-42^{\circ} 54^{\prime}$ & 1.143 & $\mathrm{Cwa}$ \\
\hline
\end{tabular}

A partir do uso do software Microsoft Office Excel versão 2013, aplicativo de planilhas eletrônicas para execução no sistema operacional Microsoft Windows, foram calculados o NHF $<7^{\circ} \mathrm{C}, \mathrm{NHF}<10^{\circ} \mathrm{C}$ e NHF $<13^{\circ} \mathrm{C}$ para todos os meses do ano e para as três localidades consideradas, sendo que os dados de NHF referentes a Antônio Dias já haviam sido determinados anteriormente por Sousa et al. (2008). Foram calculados também o valor médio da temperatura mínima e máxima de cada mês.

Os procedimentos para quantificar o NHF foram:

1 - A determinação do número de horas de frio em cada dia do período estudado;

2 - O cálculo da soma de horas de frio de cada mês;

3 - O cálculo da soma de horas de frio de cada ano.

Os valores médios mensais das temperaturas mínimas e máximas foram correlacionados com NHF $<7{ }^{\circ} \mathrm{C},<10{ }^{\circ} \mathrm{C} \mathrm{e}<13{ }^{\circ} \mathrm{C}$ para as três localidades, utilizando-se o coeficiente de correlação linear de Pearson (r), bem como avaliados o seu nível de significância a 5\% de probabilidade.

Para avaliar o comportamento médio mensal das temperaturas do ar mínimas e máximas e do NHF nas três localidades, foi ajustado o modelo proposto por (POLA et al., 1994), o qual considera a razão entre as amplitudes térmicas (RA), ou seja, a variação entre as temperaturas mínimas e máximas associada ao NHF dados por:

$$
N H F=a+b * R A+c * R A^{2}
$$

Em que NHF é o número de horas de frio abaixo de 7,2 ou $13^{\circ} \mathrm{C}$; e RA dado por:

$$
R A=24 *((K-T \text { mín }) /(T \text { máx }- \text { Tmín }))
$$

Cultura Agronômica, Ilha Solteira, v.26, n.4, p.560-572, 2017 
Em que a; b; c são coeficientes da regressão e " $\mathrm{K}$ " tem valor de 7,2 ou $13^{\circ} \mathrm{C}$, Tmín e Tmáx são as temperaturas mínimas e máximas diárias $\left({ }^{\circ} \mathrm{C}\right)$, respectivamente.

A avaliação do ajuste foi feita por meio do coeficiente de determinação $\left(R^{2}\right)$, e do erro padrão de estimativa (EPE) empregando-se a seguinte equação:

$$
E P E=\sqrt{\sum(\text { NHF observado }- \text { NHFestimado })^{2}}
$$

Em que " $n$ ” é o número de observações.

O processamento dos dados e as análises de regressões foram realizados com base no software STATISTICA ${ }^{\circledR} 7.0$ (STATSOFT, 2004).

\section{RESULTADOS E DISCUSSÃO}

Na Figura 1 são apresentadas as variações das temperaturas do ar mínima, média e máxima, em Antônio Dias, Belo Oriente e São Domingos do Prata, durante o período de junho de 2001 a dezembro de 2007.

A localidade de Antônio Dias foi a que apresentou as menores temperaturas do ar quando comparada às demais localidades, sendo que Belo Oriente foi a que apresentou as maiores temperaturas do ar, entre as três localidades. Esse fato pode estar associado à diferença de altitude entre as duas localidades, já que Belo Oriente está localizada a 233 metros de altitude enquanto Antônio Dias fica a 1.247 metros, corroborando a influência da altitude na diferença entre as temperaturas das respectivas cidades.

As temperaturas médias climatológicas das localidades de Antônio Dias, Belo Oriente, e São Domingos do Prata foram respectivamente $19,8^{\circ} \mathrm{C}, 22,6^{\circ} \mathrm{C}, 19,9^{\circ} \mathrm{C}$ (ALVARES et al., 2013), enquanto as médias observadas no período 2001 a 2007 foram $17,6^{\circ} \mathrm{C}, 23,15^{\circ} \mathrm{C}$, $18,3^{\circ} \mathrm{C}$, evidenciando que os em Antônio Dias e São Domingos do Prata as temperaturas foram mais baixas que as temperaturas das normais climatológicas. Estas variações ou reduções de temperatura média do ar em relação as normais climatológicas, podem estarem relacionadas as variações das altitudes entre as estações meteorológicas utilizadas neste estudo, ou seja, as estações das normais climatológicas estão a 761 e 720, respectivamente nos municípios de Antônio Dias e São Domingos do Prata, enquanto que as estações utilizadas neste estudo estão a 1273 e 1143, conforme dados apresentados na Tabela 1.

Verifica-se, na Figura 1a, que em Antônio Dias o verão de 2005/2006 foi o mais quente do período, enquanto que o inverno de 2004 e o de 2006 foram os mais frios durante o período avaliado, e quando compara-se com média climatológica do verão do município de Antônio Dias verifica-se que resfriamento médio no período avaliado de $2,79^{\circ} \mathrm{C}$. 


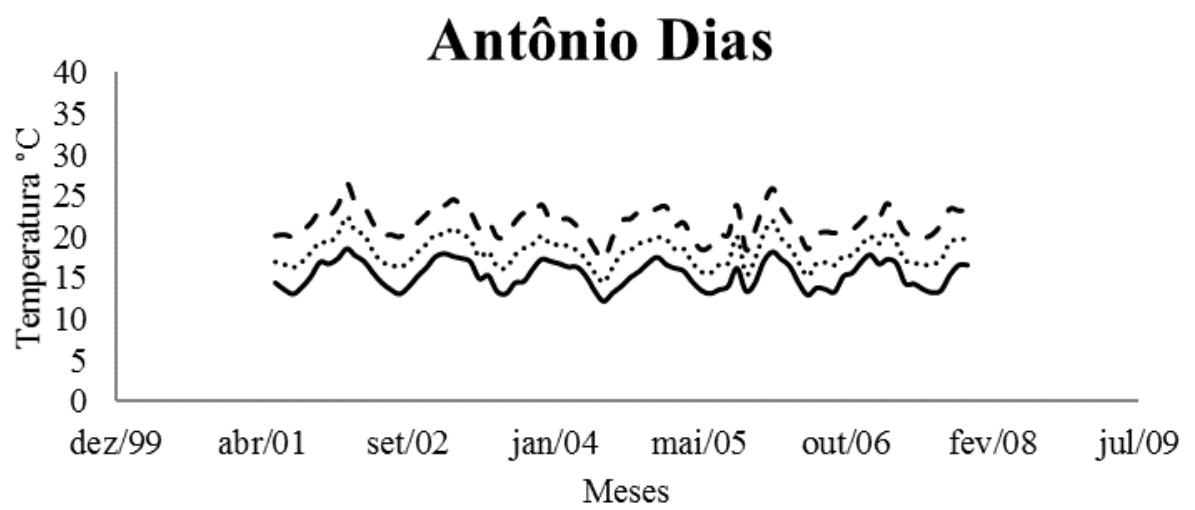

a)

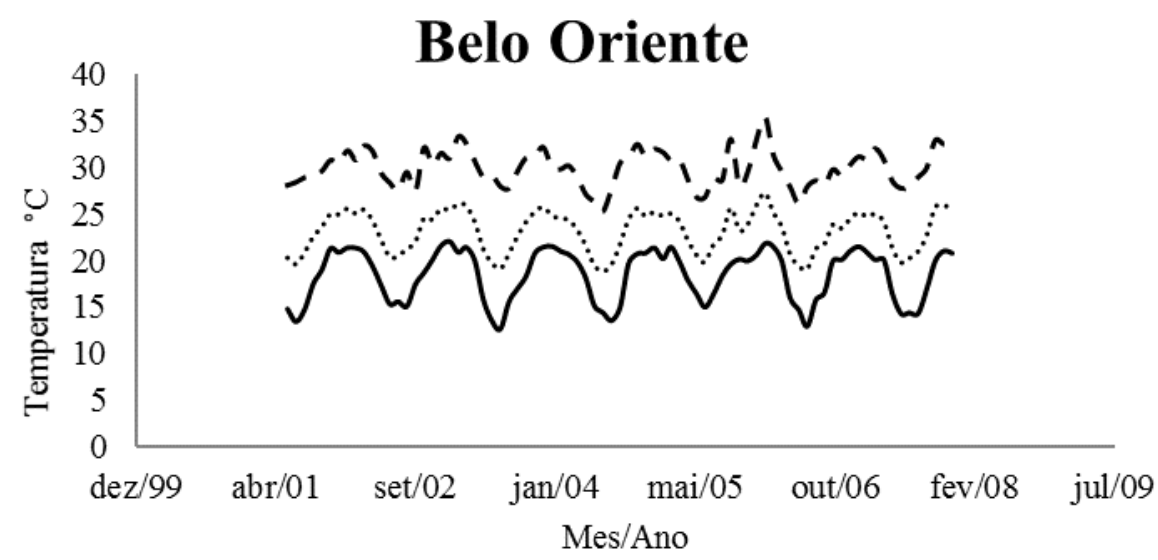

b)

\section{São Domingos do Prata}

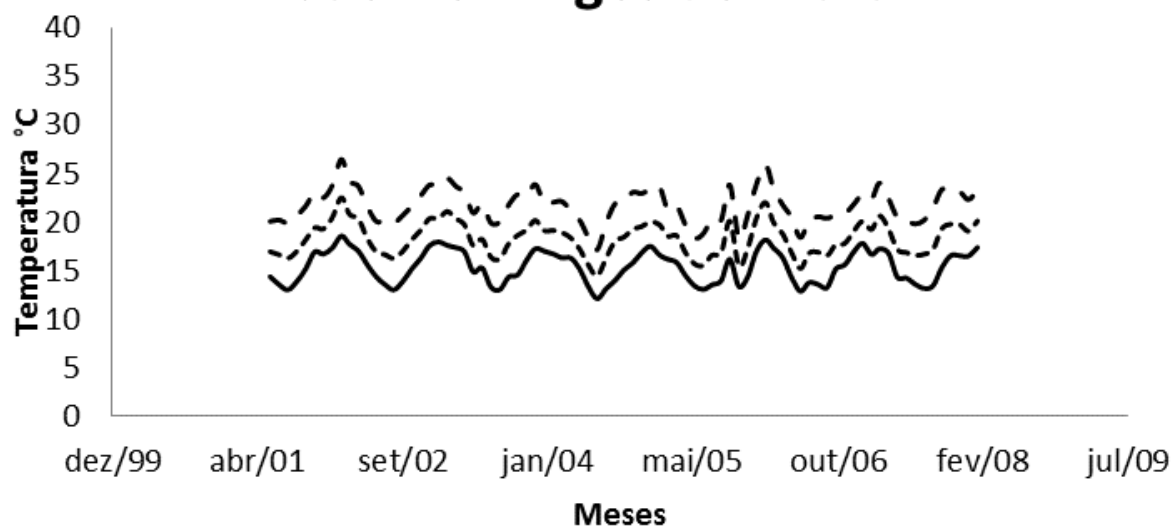

c)

-........Tempera tura Média do Ar - - - Temperatura Máxima do Ar —— Tempera tura Minima do Ar

Figura 1. Temperatura média, máxima e mínima do ar em Antônio Dias (MG) (a), em Belo Oriente (MG) (b) e em São Domingos do Prata (MG) (c). 
Na Figura 1c, pode ser observado que o verão de 2001/2002 no município de São Domingos do Prata foram os mais quentes, sendo o inverno de 2004 o mais frio do período. Para Belo Oriente na Figura 1b, percebe-se que o verão e o inverno de 2005/2006 foram os mais rigorosos bem como o inverno de 2003. Comparando-se a temperatura média do ar da normal climatológica com a temperatura média do ar observada no verão de Belo Oriente, percebe-se um desvio de $0,53^{\circ} \mathrm{C}$.

Outro ponto importante é sobre as variações das classes climatológicas entre as estações meteorológicas, considerando que as cidades são relativamente próximas, pois a maior distância observada foi entre as cidades de Antônio Dias e Belo Oriente $(57 \mathrm{~km})$ a classificação climática muda consideravelmente do clima $\mathrm{C}$ (Subtropical úmido) para o A (tropical), conforme os dados apresentados na Tabela 1. A abrangência da atuação com boa eficiência de uma estação meteorológica limita-se muitas das vezes a $150 \mathrm{~km}^{2}$ de atuação (VAREJÃO-SILVA, 2006), ou seja, pode se considerar que o raio de uma estação sobrepõe o raio da outra nos casos de Antônio Dias e São Domingos do Prata, e Belo Oriente estaria com um vazio de cobertura de apenas $30 \mathrm{~km}$ de distância em relação a São Domingos do Prata.

São apresentados na Tabela 2 os números de horas de frio médio anual para as três localidades entre o período de junho de 2001 a dezembro de 2007. Ao analisar tais dados percebe-se que o NHF $<7{ }^{\circ} \mathrm{C}$ ocorre de forma reduzida nas três localidades, ou seja, não seria necessária para a quebra de dormência ou germinação de várias espécies/cultivares, como por exemplo, o grupo de macieiras "Gala" que necessita de aproximadamente $700 \mathrm{HF}$ abaixo de $7,2^{\circ} \mathrm{C}$ (média exigência) e o grupo "Fuji" necessita entre 700 a $800 \mathrm{HF}$ (alta exigência) (PETRI et al., 2006).

Tabela 2. Número de horas de frio $<7^{\circ} \mathrm{C},<10^{\circ} \mathrm{C} \mathrm{e}<13{ }^{\circ} \mathrm{C}$ anuais para Antônio Dias, Belo Oriente e São Domingos do Prata

\begin{tabular}{cccc}
\hline Localidade & NHF $<\mathbf{7}^{\circ} \mathbf{C}$ & $\mathbf{N H F}<\mathbf{1 0}^{\circ} \mathbf{C}$ & $\mathbf{N H F}<\mathbf{1 3}^{\circ} \mathbf{C}$ \\
\hline Antônio Dias & 5,7 & 69,5 & 649 \\
Belo Oriente & 0 & 4,7 & 87,5 \\
São Domingos do Prata & 0,5 & 43 & 367,2 \\
\hline
\end{tabular}

Adicionalmente, segundo (RONQUE, 1998), para o morangueiro, as exigências vão de 380 a 700 horas acumuladas de temperaturas entre $2^{\circ} \mathrm{C}$ a $7^{\circ} \mathrm{C}$. No caso das localidades estudadas a introdução dessa espécie frutífera não é desejável, pois a mesma não se desenvolverá devido ao $\mathrm{NHF}<7^{\circ} \mathrm{C}$ não atender o mínimo necessário para que a planta se estabeleça.

Adicionalmente, observou-se também para cultura da macieira que a temperatura base inferior foi de $3^{\circ} \mathrm{C}$ para a "Fuji" e de $2^{\circ} \mathrm{C}$ para a "Gala", e por outro lado, a temperatura base superior foi de $28^{\circ} \mathrm{C}$ para a "Fuji" e de $30^{\circ} \mathrm{C}$ para a "Gala" (PUTTI et al., 2000). Para os casos das localidades avaliadas, não seria possível pois, tanto a temperatura basal inferior, quanto a superior não está adequada aos limites térmicos.

Cultura Agronômica, Ilha Solteira, v.26, n.4, p.560-572, 2017 
Por outro lado, existem culturas como as do pessegueiro e da nectarina, de ocorrência nas mais variadas condições de clima e solo, desde as localidades com inverno rigoroso (600 a $1.200 \mathrm{NHF}$ abaixo de $7,2^{\circ} \mathrm{C}$ ), até em outras praticamente desprovidas de frio hibernal, menos de $20 \mathrm{HF}$ abaixo de $7,2^{\circ} \mathrm{C}$ (BARBOSA et al., 1990) que seria possível a introdução destas culturas/variedades, nas regiões de Antônio Dias e São Domingos do Prata.

Analisando o $\mathrm{NHF}<13^{\circ} \mathrm{C}$ observa-se que a localidade de Antônio Dias apresenta grande disponibilidade deste índice, e conseguintemente algumas variedades do pessegueiro, nectarina e do kiwi, pouco exigentes em NHF (TAVARES et al., 2011), podem ser introduzidas nesse local levando-se em conta a variável disponibilidade de frio hibernal, porém as localidades de São Domingos do Prata e Belo Oriente não dispõem desta característica (PEREIRA, et al., 2002). Estes valores corroboram os encontrados por Tavares et al. (2011) na localidade de Jundiaí (SP) em que a localidade foi considerada apta para o cultivo de espécies pouco exigentes em frio hibernal.

As características fisiológicas das plantas a tolerância ao frio e a quebra da endodormencia, podem estarem associadas aos teores foliares de carboidratos não estruturais, de sacarose e de amido, pois observou-se um aumento nas plantas sob frio radicular. Estes estudos iniciaram-se recentemente e prometem serem sinalizadores de plantas resistentes/tolerantes a determinados estresses ambientais (SALES et al., 2012; ZEPPEL et al., 2011).

A distribuição mensal de NHF para as três localidades estudadas, durante o período de junho de 2001 a dezembro de 2007, pode ser observado nas Figuras 2, 3 e 4. De modo geral, a localidade de Antônio Dias apresenta os maiores valores e distribuição de horas de frio em relação às demais localidades, corroborando com os resultados apresentados na Figura 1, na qual a temperatura do ar para aquela localidade também apresentou as temperaturas mais amenas.
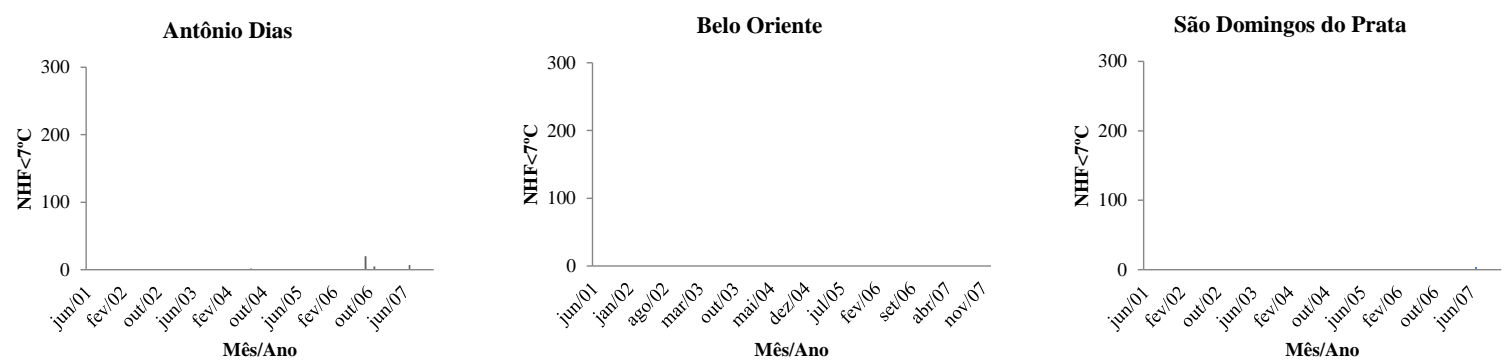

Figura 2. Número de horas de frio (NHF) menor que $7{ }^{\circ} \mathrm{C}$ acumuladas mensalmente em Antônio Dias, Belo Oriente e São Domingos do Prata, Minas Gerais. 

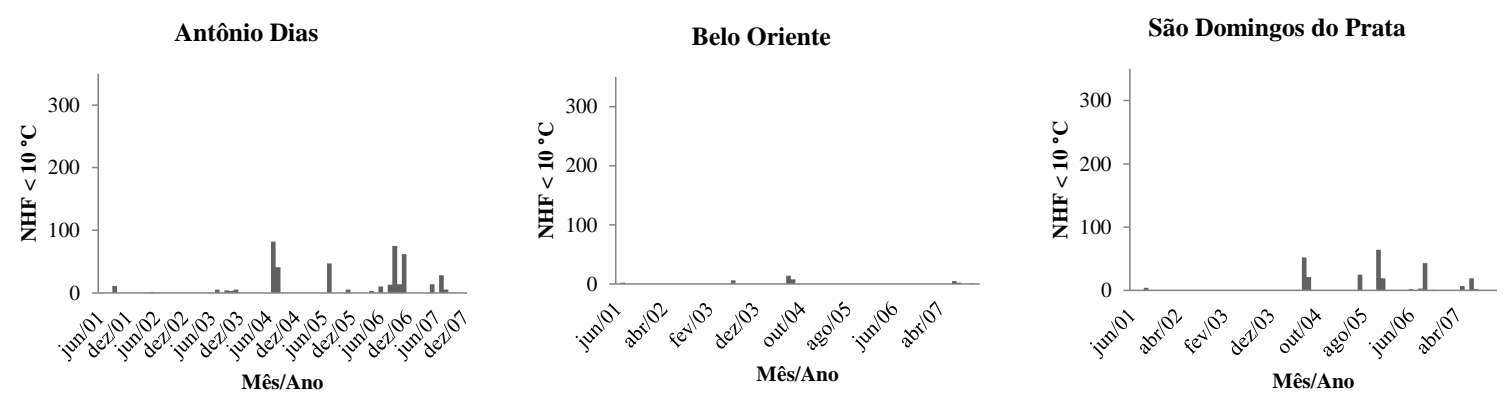

Figura 3. Número de horas de frio (NHF) menor que $10{ }^{\circ} \mathrm{C}$ acumuladas mensalmente em Antônio Dias, Belo Oriente e São Domingos do Prata, Minas Gerais.
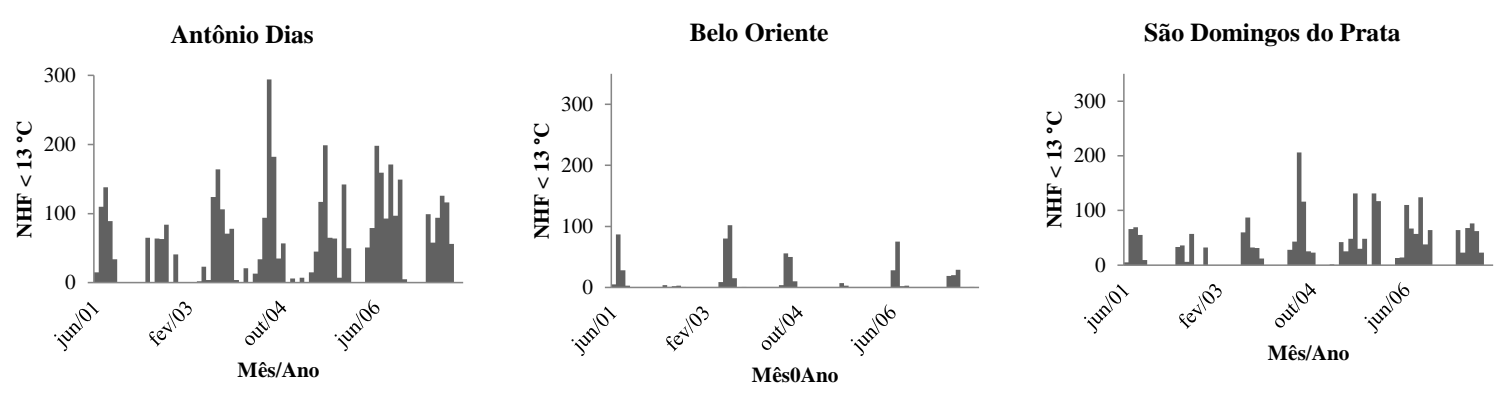

Figura 4. Número de horas de frio (NHF) menor que $13{ }^{\circ} \mathrm{C}$ acumuladas mensalmente em Antônio Dias, Belo Oriente e São Domingos do Prata, Minas Gerais.

Verifica-se, também nessa figura, a ocorrência do NHF $<13{ }^{\circ} \mathrm{C}$ em todos os anos avaliados e em todas as localidades, bem como que o ano de 2006 da localidade de Antônio Dias destaca-se com o acúmulo de $1.002 \mathrm{NHF}$ abaixo de $13{ }^{\circ} \mathrm{C}$.

Do ponto de vista de agronômico de espécies de clima temperado, em especial, as pouco exigentes em frio hibernal, a localidade de Antônio Dias e São Domingos do Prata são as mais adequadas para o cultivo destas espécies/variedades, como por exemplo, as culturas do pessegueiro e do nectarineiro, o mesmo não é verificado para a localidade de Belo Oriente.

O mesmo não acontece com NHF $<7{ }^{\circ} \mathrm{C}$ e com o NHF $<10{ }^{\circ} \mathrm{C}$ que não ocorre em todos os anos, sendo que em Belo Oriente não houve nenhuma hora abaixo de $7{ }^{\circ} \mathrm{C}$ entre o período estudado.

Pode-se notar que há uma concentração de horas de frio entre os meses de maio e novembro, meses de maior exigência para a maioria das espécies frutíferas de clima temperado (macieira, pessegueiro, marmelo entre outras). Estes resultados se assemelham aos obtidos por Cardoso et al. (2012) que observou número de horas de frio entre os meses de julho a agosto em estudos com a cultura da macieira no estado do Paraná.

A endodormência mais intensa, por exemplo, do caquizeiro 'Fuyu', ocorre no período compreendido entre a metade de maio e o início de junho em região de baixa ocorrência de frio (CARVALHO et al., 2010), e conseguintemente o pico da produção de frutos das 
espécies/cultivares de clima temperado ocorrem entre o período de outubro (PUTTI et al., 2000).

Sobre uma nova perspectiva das mudanças climáticas globais Luedeling et al. (2009) apontou que as espécies / clones das plantações florestais da Califórnia (EUA) não serão tolerantes ao aquecimento previsto para a região para o ano de 2099. Uma das propostas para mitigação deste aquecimento seria o investimento em melhoramento genético para garantir a permanência da cultura florestal nesta região.

Na Tabela 3 são apresentadas as correlações obtidas entre os NHF e as média mensais de temperaturas do ar (mínima, média e máxima) nas três cidades. Observa-se que a variável temperatura mínima média do ar apresenta maior correlação com o $\mathrm{NHF}<13{ }^{\circ} \mathrm{C}$, seguida pelo $\mathrm{NHF}<10^{\circ} \mathrm{C}$ e o $\mathrm{NHF}<7^{\circ} \mathrm{C}$.

Tabela 3. Coeficientes de Correlação de Pearson entre temperatura média mínima e máxima do ar e Número de Horas de Frio $<7{ }^{\circ} \mathrm{C},<10^{\circ} \mathrm{C}$ e $<13{ }^{\circ} \mathrm{C}$ para três localidades do Vale do Rio Doce

\begin{tabular}{ccccc}
\hline \multirow{2}{*}{ Fontes de Variação } & \multicolumn{3}{c}{ Correlação Linear de Pearson $(\mathrm{r})$} \\
\cline { 3 - 5 } & Temperatura mínima média & $-0,24 * 7{ }^{\circ} \mathrm{C}$ & $\mathrm{NHF}<10^{\circ} \mathrm{C}$ & $\mathrm{NHF}<13^{\circ} \mathrm{C}$ \\
\hline Antônio & Temper & $-0,45$ & $-0,86 *$ \\
Dias & Temperatura máxima média & $-0,15 \mathrm{n} . \mathrm{s}$ & $-0,40 *$ & $-0,77 *$ \\
\hline \multirow{2}{*}{ Belo Oriente } & Temperatura mínima média & $0 \mathrm{n} . \mathrm{s}$ & $-0,38^{*}$ & $-0,66 *$ \\
& Temperatura máxima média & $0 \mathrm{n} . \mathrm{s}$ & $-0,35 *$ & $-0,39 *$ \\
\hline \multirow{2}{*}{$\begin{array}{c}\text { São } \\
\text { Domingos } \\
\text { do Prata }\end{array}$} & Temperatura mínima média & $-0,12 \mathrm{n} . \mathrm{s}$ & $-0,40 *$ & $-0,75^{*}$ \\
\hline
\end{tabular}

*significativo a 95\% de probabilidade / n.s não significativo.

Na localidade de São Domingos do Prata e Belo Oriente, a correlação entre as variáveis $\mathrm{NHF}<7^{\circ} \mathrm{C}$ e a temperatura mínima e máxima do ar não foi significativa, possivelmente em função dessas localidades apresentarem número de horas de resfriamento reduzido ou ausente, o que dificulta o estabelecimento de relações entre as variáveis.

As relações encontradas neste estudo, corroboram os trabalhos realizados por Pedro Junior et al. (2007) na localidade de Capão Bonito (SP) e Pola et al. (1994) na região serra catarinense onde os autores verificaram a possibilidade das estimativas do $\mathrm{NHF}<7,0$ e $\mathrm{NHF}<13$, porém os mesmos não realizaram os trabalhos com o índice bioclimático $\mathrm{NHF}<$ $10^{\circ} \mathrm{C}$

Infelizmente, há um número limitado de estações meteorológicas nos quais estas variáveis são medidas de modo eficiente (FERNANDES et al., 2012), principalmente em base horária, o que demanda estações meteorológicas automáticas e que possuem custo elevado para sua aquisição e manipulação por pequenos e médios agricultores (MARIN, 2005).

Cultura Agronômica, Ilha Solteira, v.26, n.4, p.560-572, 2017 
Dessa forma, o estabelecimento de correlações e equações de regressão entre variáveis meteorológicas são de grande valia para estimar com maior facilidade variáveis com base horaria, que é o caso do NHF. Neste sentido, este estudo propôs uma gama de equações de regressão que permitam quantificá-lo utilizando variáveis climáticas com base mensal de dados (ex: valores médios mensais das temperaturas do ar mínimas e máximas), e assim facilitar o cálculo do NHF para uma dada região o que auxiliará no zoneamento agrícola de espécies/cultivares exigentes em frio hibernal.

Tendo isto em vista, para verificar a consistência das correlações obtidas, utilizou-se o modelo proposto por Pola et al. (1994) para diferentes localidades de Santa Catarina. As equações obtidas por localidade são apresentadas na Tabela 4.

Tabela 4. Equações adaptadas para Antônio Dias, Belo Oriente e São Domingos do Prata para estimar o NHF $<7{ }^{\circ} \mathrm{C},<10{ }^{\circ} \mathrm{C} \mathrm{e}<13{ }^{\circ} \mathrm{C}$, coeficiente de determinação $\left(\mathrm{R}^{2}\right)$, erro padrão de estimativa (EPE) para avaliar o desempenho do ajuste.

\begin{tabular}{cccc}
\hline Localidade & Equações & $\mathbf{R}^{2}$ & EPE \\
\hline \multirow{4}{*}{ Antônio Dias } & $\mathrm{NHF}<7{ }^{\circ} \mathrm{C}=13,4875+0,8496 * \mathrm{RA}+0,0113 * \mathrm{RA}^{2}$ & $0,12 *$ & 2,3 \\
& $\mathrm{NHF}<10^{\circ} \mathrm{C}=68,1145+6,6870 * \mathrm{RA}+0,1583 * \mathrm{RA}^{2}$ & $0,30 *$ & 13,4 \\
& $\mathrm{NHF}<13^{\circ} \mathrm{C}=110,9445+16,5845 * \mathrm{RA}+0,6252 * \mathrm{RA}^{2}$ & $0,85 *$ & 24,5 \\
\hline \multirow{3}{*}{ Belo Oriente } & $\mathrm{NHF}<7{ }^{\circ} \mathrm{C}=0+0 * \mathrm{RA}+0 * \mathrm{RA} 2$ & $0,00 \mathrm{n} . \mathrm{s}$ & 0,0 \\
& $\mathrm{NHF}<10^{\circ} \mathrm{C}=4,4107+0,4090 * \mathrm{RA}+0,0090 * \mathrm{RA}^{2}$ & $0,17 *$ & 1,8 \\
& $\mathrm{NHF}<13^{\circ} \mathrm{C}=53,1121+8,1910 * \mathrm{RA}+0,2798 * \mathrm{RA}^{2}$ & $0,64 *$ & 8,2 \\
\hline \multirow{2}{*}{$\begin{array}{c}\text { São Domingos } \\
\text { do Prata }\end{array}$} & $\mathrm{NHF}<7{ }^{\circ} \mathrm{C}=0,1578+0,0031 * \mathrm{RA}+0,00002 * \mathrm{RA}^{2}$ & $0,01 \mathrm{~ns}$ & 0,3 \\
& $\mathrm{NHF}<10^{\circ} \mathrm{C}=28,9983+2,1345 * \mathrm{RA}+0,0385 * \mathrm{RA}^{2}$ & $0,13 *$ & 10,5 \\
& $\mathrm{NHF}<13^{\circ} \mathrm{C}=89,7073+12,2974 * \mathrm{RA}+0,4180 * \mathrm{RA}^{2}$ & $0,68 *$ & 23,9 \\
\hline
\end{tabular}

*significativo a 5\% de probabilidade / n.s não significativo.

Ao analisar a Tabela 4 percebe-se que o desempenho do ajuste das equações segue a mesma tendência das correlações obtidas, ou seja, o NHF $<13{ }^{\circ} \mathrm{C}$ é melhor explicado pela variável RA, que relaciona as variáveis temperaturas mínimas e máximas do ar (nesse modelo estas temperaturas são utilizadas para quantificar a amplitude térmica), seguidas pelo NHF $<10{ }^{\circ} \mathrm{C}$ e NHF $<7{ }^{\circ} \mathrm{C}$. Nota-se também que as localidades com a maior quantidade de horas de frio acumuladas permitem melhores estimativas do NHF, comprovado pelo coeficiente de determinação da localidade de Antônio Dias quando comparado às demais localidades.

De acordo com os valores do coeficiente de determinação $\left(\mathrm{R}^{2}\right)$ do $\mathrm{NHF}<13^{\circ} \mathrm{C}$, percebe-se que modelo proposto por Pola et al. (1994) apresentou estimativas fortes a moderadas para o NHF $<13{ }^{\circ} \mathrm{C}$ para as três localidades (PIMENTEL-GOMES, 1987; MUKAKA, 2012). Já para o NHF $<10{ }^{\circ} \mathrm{C} \mathrm{e}<7{ }^{\circ} \mathrm{C}$ o modelo não se mostrou eficiente nas estimativas, apresentando coeficientes de regressão muito fracos, proem significativos, principalmente nas localidades em que ocorreram poucas horas de frio. 


\section{CONCLUSÃO}

As variáveis número de horas de frio menor que $13^{\circ} \mathrm{C}$ correlacionaram-se bem com a média mensal das temperaturas mínimas e máximas do ar nas três localidades estudadas.

$\mathrm{O}$ mesmo não aconteceu com o número de horas de frio menor que $7^{\circ} \mathrm{C}$ e $10^{\circ} \mathrm{C}$, que não apresentou correlação significativa na localidade de Belo Oriente, e ainda valores muito fracos de coeficiente de determinação na localidade de Antônio Dias e São Domingos do Prata.

Antônio Dias e São Domingos do Prata apresentaram características térmicas adequadas para o cultivo de espécies frutíferas de clima temperado pouco exigentes em frio hibernal, enquanto a localidade de Belo Oriente mostrou-se inadequada para o cultivo das mesmas.

\section{AGRADECIMENTOS}

Os autores agradecem ao Conselho Nacional de Desenvolvimento Científico e Tecnológico ( $\mathrm{CNPq})$, que valoriza o despertar e a criação científica nas academias de nosso país; bem como a engenheira agrônoma Francine Aparecida Sousa pelo processamento de parte dos dados, e agradecem ainda à empresa florestal Celulose Nipo-Brasileira (CENIBRA), pelo fornecimento dos mesmos.

\section{REFERÊNCIAS BIBLIOGRÁFICAS}

ALVARES, C. A.; STAPE, J. L.; SENTElhas, P. C.; GONÇAlVES, J. L. M.; SPAROVEK, G. Köppen's climate classification map for Brazil. Meteorologische Zeitschrift, Stuttgart, v. 22, n. 6, p.711-728, 2013.

ASSAD, E. D.; PINTO, H. S.; ZULLO JÚNIOR, J.; ÁVILA, A. M. H. Impacto das mudanças climáticas no zoneamento agroclimático do café no Brasil. Pesquisa Agropecuária Brasileira, Brasília, v. 39, n. 11, p.1057-1064, 2004.

BARBOSA, W.; CAMPO-DALL'ORTO, F. A.; OJIMA, M.; SAMPAIO, V. R.; BANDEL, G. Ecofisiologia do desenvolvimento vegetativo e reprodutivo do pessegueiro em localidade subtropical. Campinas, SP: IAC, 1990. 37 p. (Documentos IAC, 17)

CARDOSO, L. S.; BERGAMASCHI, H.; BOSCO, L. C.; DE PAULA, V. A.; MARODIN, G. A. B.; CASAMALI, B.; NACHTIGALL, G. R. Climate availability for apple trees in Vacaria, southern Brazil. Ciência Rural, Santa Maria, v. 42, n. 11, p.1960-1967, 2012.

CARVALHO, R. I. N.; BIASI, L. A.; ZANETTE, F.; RENDOKE, J. C.; SANTOS, J. M.; PEREIRA, G. P. Dinâmica da dormência de gemas de caquizeiro 'Fuyu' em região de baixa ocorrência de frio. Scientia Agraria, Curitiba, v. 11, n. 1, p.57-63, 2010. 
FERNANDES, D. S.; HEINEMANN, A. B; PAZ, R. L. F.; AMORIM, A. O. Regional and local calibration of Hargreaves equation for estimating reference evapotranspiration. Revista Ciência Agronômica, Fortaleza, v. 43, n. 2, p.246-255, 2012.

LUEDELING, E.; ZHANG, M.; GIRVETZ, E. H. Climatic changes lead to declining winter chill for fruit and nut trees in California during 1950-2099. PLoS One, San Francisco, v. 4, n. 1, p.7-10, 2009.

MARIN, F. R. Introdução ao uso de Sistemas Automáticos de Aquisição de dados na Agrometeorologia. 1. ed. Campinas: Embrapa Informática Agropecuária, 2005. 100 p. (Documentos - INFOTECA-E)

MUKAKA, M. M. A Guide to Appropriate Use of Correlation Coefficient in Medical Research. Malawi Medical Journal: The Journal of Medical Association of Malawi, Malawi, v. 24, n. 3, p.69-71, 2012.

MORAIS, H.; CARBONIERI, J. Chilling hours and units in apple orchards with distinct microclimate. Revista Brasileira de Fruticultura, Jaboticabal, v. 37, n. 1, p.1-12, 2015.

PEDRO JUNIOR, M. J.; BARBOSA, W.; ROLIM, G. S.; CASTRO, J. L. Época de florescimento e horas de frio para pessegueiros e nectarineiras. Revista Brasileira de Fruticultura, Jaboticabal, v. 29, n. 3, p.425-430, 2007.

PEREIRA, A. R.; ANGELOCCI, L. R.; SENTELHAS, P. C. Agrometeorologia fundamentos e aplicações práticas. 1. ed. Guaíba: Agropecuária, 2002. 478 p.

PETRI, J. L. Formação de flores, polinização e fertilização. In: EPAGRI. A cultura da macieira. 1. ed. Florianópolis: Epagri, 2006. p. 229-260.

PIMENTEL-GOMES, F. Curso de estatística experimental. 12. ed. São Paulo: Nobel, 1987. $466 \mathrm{p}$.

POLA, A. C.; BLEICHER, J; BERNARDI, J. Avaliação de modelo de unidades e horas de frio para a previsão do início de brotação em macieira, cv. Gala. Revista Brasileira de Fruticultura, Cruz de Almas, v. 16, n. 1, p.105-118, 1994.

PUTTI, G. L.; MENDEZ, M. E.; PETRI, J. L. Unidades de frio e de calor para a brotação de macieira (Malus domestica, Borck.), 'Gala' e 'Fuji'. Revista Brasileira de Agrociência, Pelotas, v. 6, n. 3, p.194-196, 2000.

RIBEIRO, R. V.; MACHADO, E. C.; BRUNINI, O. Ocorrência de condições ambientais para a indução do florescimento de laranjeiras no Estado de São Paulo. Revista Brasileira de Fruticultura, Jaboticabal, v. 28, n. 2, p.247-253, 2006.

RONQUE, E. R. V. Cultura do morangueiro: revisão e prática. 1. ed. Curitiba: EMATER, 1998. 206 p.

Cultura Agronômica, Ilha Solteira, v.26, n.4, p.560-572, 2017 
SALES, C. R. G.; RIBEIRO, R. V.; MACHADO, D. F. S. P; MACHADO, R. S.; DOVIS, V. L.; LAGÔA, A. M. M. A. Gas exchange and carbohydrate balance in sugarcane plants under root stressful conditions. Bragantia, Campinas, v. 71, n. 3, p.319-327, 2012.

SILVA, J. B.; HERTER, F. G.; PAZ, S. R. Disponibilidade das horas de frio $\left(\mathrm{hf} \leq 7,2^{\circ} \mathrm{C}\right)$ na Encosta da Serra do Sudeste. Pelotas, RS, Revista Brasileira de Agrometeorologia, Santa Maria, v. 10, n. 1, p.113-122, 2002.

SOUSA, F. A.; SOUZA, M. J. H.; RIBEIRO, A.; LEITE, F. P.; MOURA, V. V. Número de horas de frio em Cocais, Minas Gerais. In: CONGRESSO BRASILEIRO DE METEOROLOGIA, 15., 2008, São Paulo. Anais... São Paulo. Sociedade Brasileira de Agrometeorologia, 2008. p. 63-67.

SOUZA, M. J. H.; SILVA, V. E.; NOGUEIRA, G. S.; RIBEIRO, A.; LEITE, F. P. Estudo das Correlações entre Temperatura Mínima e Máxima do Ar e do Número de Horas de Frio em Três Localidades de Minas Gerais. In: CONGRESSO BRASILEIRO DE AGrometeOrologiA, 16., 2009, Belo Horizonte. Anais... Belo Horizonte: Sociedade Brasileira de Agrometeorologia, 2009. p. 25-29.

STATSOFT I. Statistica 7.0 para Windows: StatSoft, Inc., Tulsa, USA, 1984-2004. CD ROM.

TAVARES, P. S.; GIAROLlA, A.; CHOU, C. S Estimativa do Número de Horas de Frio em Jundiaí-SP para Fruticulturas de Clima Temperado, Com Base Nas Previsões Sazonais Do Modelo Regional Eta. In: CONGRESSO BRASILEIRO DE AGROMETEOROLOGIA, 17., 2011, Guarapari. Anais... Guarapari: Sociedade Brasileira de Agrometeorologia, 2011. p. 100-105.

VAREJÃO-SILVA, M. A. Meteorologia e climatologia. Recife: Versão Digital, 2006. Disponível em: http://www.agritempo.gov.br/publish/publicacoes/livros/METEROLOGIA_E_CLIMATOL OGIA_VD2_Mar_2006.pdf. Acesso: 13 jul. 2017.

ZEPPEL, M. J. B.; ADAMS, H. D.; ANDEREGG, W. R. L. Mechanistic causes of tree drought mortality: recent results, unresolved questions and future research needs. New Phytologist, Hoboken, v. 192, n. 4, p.800-803, 2011. 